\title{
Turnpike properties and strict dissipativity for discrete time linear quadratic optimal control problems
}

\author{
Lars Grüne \\ Mathematical Institute \\ University of Bayreuth \\ 95440 Bayreuth, Germany \\ lars.gruene@uni-bayreuth.de
}

\author{
Roberto Guglielmi \\ Johann Radon Institute \\ Altenberger Straße 69 \\ 4040 Linz, Austria \\ roberto.guglielmi@ricam. oeaw.ac.at
}

March 30, 2017

\begin{abstract}
We investigate turnpike behaviour of discrete time optimal control problems with linear dynamics and linear-quadratic cost functions including state and control constraints. We give necessary and sufficient conditions in terms of spectral criteria and matrix inequalities. As important tools we use the concepts of strict dissipativity and a new property called strict pre-dissipativity of a system at an equilibrium point and link these properties to the turnpike behaviour of the optimal control problem. Moreover, we give further conditions to ensure that the turnpike behavior is of exponential type, i.e., the optimal trajectories are exponentially close to a steady-state of the system for all but finitely many time instants whose number is bounded independently of the optimization horizon.
\end{abstract}

MSC Classification: 49K15, 49N10, 49J15, 93D20, 93C15

Keywords: turnpike property, linear-quadratic optimal control, dissipativity, detectability, Lyapunov matrix inequality, long time behaviour

\section{Introduction}

The turnpike property describes the fact that trajectories of optimally controlled systems "most of the time" stay close to an equilibrium point. It has been described already in the early days of optimal control by von Neumann [17] and in the book by Dorfman, Samuelson and Solow [7]. In this book also the name "turnpike property" has been introduced, because of the similarity of the optimal trajectories with driving a car from a point A to a point B using a turnpike or highway. Such a tour consists of three phases: driving to the highway (i.e., approaching the equilibrium), driving on the highway (i.e., staying near the equilibrium) and leaving the highway (i.e., moving away from the equilibrium). Turnpike phenomena have attracted the attention of researchers because of the structural insights they allow on the structure of the optimal solutions, particularly in mathematical economy, see, e.g., [12], but also as a method for synthesizing long term optimal trajectories $[1,13,16]$ and in recent years for analyzing model predictive control schemes [9], [11, Chapter 8]. In 
this paper we investigate this property for optimal control problems with linear dynamics and a cost function consisting of quadratic, linear and constant terms. Here the cost is convex but not necessarily strictly convex, i.e., the quadratic term in the cost is positive semidefinite but not necessarily positive definite. In this context, [13] (in continuous time) and [6] (in discrete time) formulate sufficient conditions for the turnpike property to hold, even in an exponential form. The former reference presents an observability condition ([13, Theorem 1.1]) while the latter uses a condition on the spectrum of the matrix $A$ defining the dynamics ([6, Proposition 4.5]). The main results in this paper unify these two conditions, cf. Remark 8.5, below. We note that we present these results for discrete time systems in this paper, but we expect that the derivation of their continuous time analogues is an easy exercise.

It is known since the pioneering work of Willems $[18,19,20]$ that there is a strong relation between the long time behavior of optimally controlled systems and dissipativity and strict dissipativity properties introduced in Willems' papers. For the turnpike property discussed above, this relation was worked out in detail in [10] for nonlinear discrete time problems, although partial results in this direction are already much older, see, e.g., [4, Theorem 4.2]. In this paper, we will on the one hand use strict dissipativity as a sufficient condition for the turnpike property. On the other hand, we will derive new characterizations for strict dissipativity and for the newly introduced property of strict pre-dissipativity in terms of the system matrices for the linear quadratic problems under consideration, which go beyond the nonlinear results from [10]. These will then also lead to new necessary conditions for the turnpike properties under consideration.

One of the key novelties which distinguishes the results in the present paper from earlier ones on linear quadratic optimal control problems is the consideration of state and input constraints. There are (at least) three conceptually different situations how the turnpike property interacts with state constraints: In the first case the turnpike phenomenon occurs both with and without constraints, provided the turnpike equilibrium lies inside the set of admissible states and controls. The second situation is that the turnpike phenomenon only occurs if state constraints are present, but the location of the turnpike equilibrium is independent of the particular form of the constraints. Finally, in the third situation the position of the turnpike equilibrium depends on the constraint sets. In this paper we investigate the first and the second situation, while the third will be addressed in future research.

The remainder of the paper is organized as follows. In Section 2 we describe the optimal control problem we study and define the turnpike properties and the dissipativity properties considered in this paper. Section 3 summarizes (and slightly extend) known results which show that strict (pre)-dissipativity implies turnpike properties. Section 4 introduces a matrix inequality charecterization of strict (pre)-dissipativity. Sections 5 and 6 reformulates this inequality in terms of the system matrices. Section 7 presents results showing that turnpike properties imply strict (pre)-dissipativity. Finally, the main results and some examples are collected in Section 8. A technical auxiliary result is stated and proved in the Appendix. 


\section{Setting and preliminaries}

We consider discrete-time linear quadratic optimal control problems

$$
\underset{u \in \mathbb{U}^{N}\left(x_{0}\right)}{\operatorname{minimize}} J_{N}\left(x_{0}, u\right)
$$

where

$$
\begin{gathered}
J_{N}\left(x_{0}, u\right):=\sum_{k=0}^{N-1} x(k)^{T} Q x(k)+u(k)^{T} R u(k)+s^{T} x(k)+v^{T} u(k)+c, \\
x(k+1)=A x(k)+B u(k), \quad x(0)=x_{0},
\end{gathered}
$$

$N \in \mathbb{N}, x(k) \in \mathbb{R}^{n}, u(k) \in \mathbb{R}^{m}, A \in \mathbb{R}^{n \times n}, B \in \mathbb{R}^{n \times m}, Q \in \mathbb{R}^{n \times n}$ and $R \in \mathbb{R}^{m \times m}$, with $Q$ and $R$ symmetric, $Q \geq 0$ and $R>0$, and $s \in \mathbb{R}^{n}, v \in \mathbb{R}^{m}, c \in \mathbb{R}, x_{0} \in \mathbb{R}^{n}$.

In what follows, we consider the optimal control problem (2.1) under input constraints $\mathbb{U} \subset \mathbb{R}^{m}$ and state constraints $\mathbb{X} \subseteq \mathbb{R}^{n}$, with both $\mathbb{X}$ and $\mathbb{U}$ being closed sets. To this end, for each $x_{0} \in \mathbb{X}$ we define the space of admissible controls

$$
\mathbb{U}^{N}\left(x_{0}\right):=\left\{u \in \mathbb{U}^{N} \mid x_{u}\left(k, x_{0}\right) \in \mathbb{X} \text { for all } k=0, \ldots, N\right\} .
$$

Here and in the following we denote by $x_{u}\left(\cdot, x_{0}\right)$ the solution of $(2.2)$ with control $u$ and initial value $x_{0}$.

We abbreviate the dynamics $f(x, u):=A x+B u$ and the stage cost as

$$
\ell(x, u):=x^{T} Q x+u^{T} R u+s^{T} x+v^{T} u+c .
$$

We define the optimal value function

$$
V_{N}\left(x_{0}\right):=\inf _{u \in \mathbb{U}^{N}\left(x_{0}\right)} J_{N}\left(x_{0}, u\right)
$$

and we call a control sequence $u^{\star}(\cdot)$ and corresponding trajectory $x^{\star}(\cdot, x)$ optimal, if $J_{N}\left(x, u^{\star}\right)=V_{N}(x)$ holds. Moreover, we say that $\left(x^{e}, u^{e}\right) \in \mathbb{X} \times \mathbb{U}$ is an equilibrium for the dynamics $f$ if it satisfies $f\left(x^{e}, u^{e}\right)=x^{e}$.

Definition 2.1: (i) We say that the optimal control problem (2.1)-(2.2) has the turnpike property at an equilibrium $\left(x^{e}, u^{e}\right) \in \mathbb{X} \times \mathbb{U}$ on a set $\mathbb{X}_{t p} \subset \mathbb{X}$, if for each compact set $K \subset \mathbb{X}_{t p}$ and for each $\varepsilon>0$ there exists a constant $C_{K, \varepsilon}>0$ such that for all $x \in K$ and all $N \in \mathbb{N}$ the optimal trajectories $x^{\star}(k, x)$ of (2.1) with initial value $x$ satisfy

$$
\#\left\{k \in\{0, \ldots, N\} \mid\left\|x^{\star}(k, x)-x^{e}\right\|>\varepsilon\right\} \leq C_{K, \varepsilon} .
$$

(ii) We say that the optimal control problem (2.1)-(2.2) has the near equilibrium turnpike property at an equilibrium $\left(x^{e}, u^{e}\right) \in \mathbb{X} \times \mathbb{U}$, if for each $R>0, \varepsilon>0$ and $\delta>0$ there exists a constant $C_{R, \varepsilon, \delta}>0$ such that for all $x \in \mathbb{X}$ with $\left\|x-x^{e}\right\| \leq R$, all $N \in \mathbb{N}$, and all trajectories $x_{u}(k, x)$ satisfying $J_{N}(x, u) \leq N \ell\left(x^{e}, u^{e}\right)+\delta$ for some $u \in \mathbb{U}$, the inequality

$$
\#\left\{k \in\{0, \ldots, N\} \mid\left\|x_{u}(k, x)-x^{e}\right\|>\varepsilon\right\} \leq C_{R, \varepsilon, \delta} .
$$

holds. 
Here $\# A$ denotes the number of elements of the set $A$. In words, these properties state that the optimal/near equilibrium trajectories stay in an $\varepsilon$-neighbourhood of $x^{e}$ for all but finitely many "exceptional" time instants whose number is bounded independently of the optimization horizon $N$.

Remark 2.2: (i) If the equilibrium $\left(x^{e}, u^{e}\right)$ lies in the interior of $\mathbb{X} \times \mathbb{U}$, then Definition 2.1(i) implies that $(A, B)$ is stabilizable, because otherwise there would be initial conditions $\bar{x}_{0}$ arbitrarily close to 0 with $\left\|x_{u}\left(k, \bar{x}_{0}\right)\right\| \geq \varepsilon_{x}>0$ for all $k \in \mathbb{N}$ and $\varepsilon_{x}>0$ independent of the control sequence $u$. This would imply the existence of $x_{0}:=\bar{x}_{0}+x^{e} \in \mathbb{X}$ (sufficiently close to $x^{e}$ ) such that $\left\|x_{u}\left(k, x_{0}\right)-x^{e}\right\|=\left\|x_{u-u^{e}}\left(k, \bar{x}_{0}\right)\right\| \geq \varepsilon_{x}>0$ for all control sequences $u \in \mathbb{U}$ and all $k \in \mathbb{N}$, which contradicts the turnpike property.

(ii) In contrast to (i), Definition 2.1(ii) does not imply stabilizability, because there may not be nontrivial trajectories other than $x(k) \equiv x^{e}, u(k) \equiv u^{e}$ satisfying the assumed inequality for $J_{N}$. A simple example for such a system is $x(k+1)=x(k)$ with $\ell(x, u)=x^{2}$ and $x^{e}=u^{e}=0$.

(iii) If $(A, B)$ is stabilizable, then Definition 2.1(ii) implies Definition 2.1(i) provided $\left(x^{e}, u^{e}\right)$ lies in the interior of $\mathbb{X} \times \mathbb{U}$. This is because stabilizability implies the existence of a stabilizing feedback law $F$ such that the control $u(k)=F\left(x(k)-x^{e}\right)+u^{e}$ yields $x_{u}\left(k, x_{0}\right) \rightarrow x^{e}$ exponentially fast and $x_{u}\left(k, x_{0}\right) \in \mathbb{X}, u(k) \in \mathbb{U}$ for all $k \in \mathbb{N}$ if $x_{0}$ lies in a sufficiently small neighbourhood $\mathcal{N}$ of $x^{e}$. This implies the existence of $C>0$ with $V_{N}(x) \leq N \ell\left(x^{e}, u^{e}\right)+C$ for all $x \in \mathcal{N}$. Hence, choosing $\mathbb{X}_{t p}=\mathcal{N}$, all optimal trajectories starting in $\mathbb{X}_{t p}$ satisfy the conditions of Definition 2.1(ii) and thus the turnpike property holds.

(iv) If $\mathbb{X} \times \mathbb{U}$ is convex with nonvoid interior, then we can find a larger set $\mathbb{X}_{t p}$ as in (iii). More precisely, let $\mathcal{R}$ denote the set of initial conditions which can be controlled into the set $\mathcal{N}$ from (iii). Then a modification of the proof of [3, Proposition 12] shows that for each compact subset $K \subset$ int $\mathcal{R}$ there is $C_{K}>0$ with $V_{N}(x) \leq N \ell\left(x^{e}, u^{e}\right)+C_{K}$ for all $x \in K$ and $N \in \mathbb{N}$. Hence, using the same argument as in (iii), the set $\mathbb{X}_{t p}$ can be chosen as int $\mathcal{R}$.

(v) The statement from (iii) remains true in case $\left(x^{e}, u^{e}\right) \in \partial(\mathbb{X} \times \mathbb{U})$ if for each $x \in \mathbb{X}$ sufficiently close to $x^{e}$ there exists an admissible control $u_{x}$ with $x_{u_{x}}(k, x) \rightarrow x_{e}$ and $u_{x}(k) \rightarrow u^{e}$, both exponentially fast. However, in contrast to (iii), for $x^{e}$ or $u^{e}$ not lying in the interior of the respective constraint set, the existence of such a $u_{x}$ cannot in general be concluded from stabilizability of $(A, B)$.

In simple words, part (iii) of the remark shows that the near equilibrium turnpike property plus stabilizability implies the turnpike property.

So far we have not specified how fast the number $C_{K, \varepsilon}$ in the turnpike property grows if $\varepsilon \rightarrow 0$, or, equivalently, how fast $\varepsilon>0$ shrinks when we allow $C_{K, \varepsilon}$ to grow (always for fixed compact set $K \subset \mathbb{X}_{t p}$ ). A variant of the turnpike which takes this aspect onto account is the following exponential form of the turnpike property.

Definition 2.3: We say that the turnpike property from Definition 2.1(i) is exponential, if there is $\theta \in(0,1)$ such that for each compact set $K \subset \mathbb{X}_{t p}$ there is a constant $m_{K}>0$ such that $C_{\varepsilon, K}$ can be chosen as

$$
C_{\varepsilon, K} \leq m_{K}+\log _{\theta} \varepsilon .
$$


We note that an equivalent inequality is $\varepsilon \leq M_{K} \theta^{C_{\varepsilon, K}}$ with $M_{K}=\theta^{m_{K}}$. This shows that $\varepsilon$ shrinks exponentially fast when the number of exceptional points $C_{\varepsilon, K}$ grows. With this formulation it is easily checked that Definition 2.3 is equivalent to [6, Definition 2.2(ii)] when setting $\varepsilon=\sigma_{P}(N)$ and $C_{\varepsilon, R}=N-P$.

Our goal in this paper is to find easily checkable conditions on the data of the optimal control problem (2.1)-(2.2) (i.e., on $A, B, Q, R, s, v$ and $c$ ) under which we can guarantee that turnpike properties hold. The next definitions provide the key concepts we use for this goal. For the definitions we recall that

$$
\mathcal{K}:=\left\{\alpha: \mathbb{R}_{0}^{+} \rightarrow \mathbb{R}_{0}^{+}: \alpha \text { continuous, strictly increasing with } \alpha(0)=0\right\} .
$$

Definition 2.4: (i) We call the LQ problem strictly pre-dissipative at an equilibrium $\left(x^{e}, u^{e}\right)$ on sets $X \subseteq \mathbb{R}^{n}, U \subseteq \mathbb{R}^{m}$ if there exist a storage function $\lambda: X \rightarrow \mathbb{R}$ which is bounded on bounded subsets of $X$, a function $\alpha \in \mathcal{K}$ and an equilibrium $\left(x^{e}, u^{e}\right) \in \mathbb{R}^{n} \times \mathbb{R}^{m}$ which satisfy the inequality

$$
\lambda(f(x, u)) \leq \lambda(x)+\ell(x, u)-\ell\left(x^{e}, u^{e}\right)-\alpha\left(\left\|x-x^{e}\right\|\right)
$$

for all $x \in X$ and all $u \in U$ with $f(x, u) \in X$.

(ii) The system is called strictly dissipative at an equilibrium $\left(x^{e}, u^{e}\right)$ on sets $X \subseteq \mathbb{R}^{n}$, $U \subseteq \mathbb{R}^{m}$ if it is strictly pre-dissipative in the sense of (i) and $\lambda$ is bounded from below on $X$.

We note that strict pre-dissipativity on a set $X$ implies that the problem is strictly dissipative on each bounded subset $\widetilde{X}$ of $X$. Moreover, it is straightforward to see that strict pre-dissipativity holds if and only if the following modified cost function

$$
\tilde{\ell}(x, u):=\ell(x, u)-\ell\left(x^{e}, u^{e}\right)+\lambda(x)-\lambda(f(x, u))
$$

satisfies

$$
\tilde{\ell}(x, u) \geq \alpha\left(\left\|x-x^{e}\right\|\right)
$$

for all $x \in X$ and $u \in U$.

\section{Strict (pre-)dissipativity implies turnpike}

In this section we present (and slightly extend) known results which show that strict dissipativity implies turnpike properties. We start with the near equilibrium turnpike property.

Theorem 3.1: Consider the LQ-problem (2.1), (2.2) with state and control constraint sets $\mathbb{X} \subseteq \mathbb{R}^{n}$ and $\mathbb{U} \subseteq \mathbb{R}^{m}$. Assume that

(i) the problem is strictly dissipative at an equilibrium $\left(x^{e}, u^{e}\right)$ or

(ii) the problem is strictly pre-dissipative at an equilibrium $\left(x^{e}, u^{e}\right)$ and $\mathbb{X}$ is bounded.

Then the near equilibrium turnpike property holds at $\left(x^{e}, u^{e}\right)$. 
Proof. (i) follows from [8, Theorem 5.3] and (ii) follows from (i) since strict pre-dissipativity with bounded $\mathbb{X}$ implies strict dissipativity.

The following Corollary extends this to the optimal turnpike property, where we need the additional assumptions that $(A, B)$ is stabilizable and $\left(x^{e}, u^{e}\right)$ lies in the interior of $\mathbb{X} \times \mathbb{U}$.

Corollary 3.2: Consider the LQ-problem (2.1), (2.2) with state and control constraint sets $\mathbb{X} \subseteq \mathbb{R}^{n}$ and $\mathbb{U} \subseteq \mathbb{R}^{m}$. Assume that $(A, B)$ is stabilizable and

(i) the problem is strictly dissipative at an equilibrium $\left(x^{e}, u^{e}\right) \in \operatorname{int}(\mathbb{X} \times \mathbb{U})$ or

(ii) the problem is strictly pre-dissipative at an equilibrium $\left(x^{e}, u^{e}\right) \in \operatorname{int}(\mathbb{X} \times \mathbb{U})$ and $\mathbb{X}$ is bounded.

Then the turnpike property holds at $\left(x^{e}, u^{e}\right)$.

Proof. It follows immediately from Theorem 3.1 and Remark 2.2(iii).

We finally show that strict (pre-)dissipativity also implies the exponential turnpike property, under an additional convexity assumption on $\mathbb{X} \times \mathbb{U}$.

Theorem 3.3: Consider the LQ-problem (2.1), (2.2) with state and control constraint sets $\mathbb{X} \subseteq \mathbb{R}^{n}$ and $\mathbb{U} \subseteq \mathbb{R}^{m}$. Assume that $(A, B)$ is stabilizable, that $\mathbb{X} \times \mathbb{U}$ is convex and that

(i) the problem is strictly dissipative at an equilibrium $\left(x^{e}, u^{e}\right) \in \operatorname{int}(\mathbb{X} \times \mathbb{U})$ or

(ii) the problem is strictly pre-dissipative at an equilibrium $\left(x^{e}, u^{e}\right) \in \operatorname{int}(\mathbb{X} \times \mathbb{U})$ and $\mathbb{X}$ is bounded,

in either case with a storage function $\lambda(x)=x^{T} P x+q^{T} x$ for some $P \in \mathbb{R}^{n \times n}$ and $q \in \mathbb{R}^{n}$. Then the exponential turnpike property holds at $\left(x^{e}, u^{e}\right)$.

Proof. It is sufficient to prove (i) because then (ii) follows immediately. We choose $\mathbb{X}_{t p}$ as in Remark 2.2(iv) and use [6, Theorem 5.6] with $\mathbb{X}_{0}=K \subset \mathbb{X}_{t p}$. This theorem yields the exponential turnpike property under three conditions (i), (ii) and (iii-a). Condition (i) of this theorem follows from (2.4) and the fact that for the assumed form of $\lambda$ the modified $\operatorname{cost} \tilde{\ell}$ is again linear-quadratic. In case $\mathbb{X}$ is compact, Condition (ii) follows from $[6$, Proposition 6.4], where the proof of [6, Proposition 6.1] restricted to a ball around $\left(x^{e}, u^{e}\right)$ contained in $\mathbb{X} \times \mathbb{U}$ can be used in order to establish condition (b) of [6, Proposition 6.4]. In case $\mathbb{X}$ is compact, condition (iii-a) of [6, Theorem 5.6] holds and the exponential turnpike property follows.

In case $\mathbb{X}$ is unbounded, an inspection of the proofs of [6, Proposition 6.4] and [6, Theorem 5.6] shows that the respective assertions remain true if compactness of $\mathbb{X}$ is replaced by the following property: for each compact set $\mathbb{X}_{0} \subset \mathbb{X}_{t p}$ there exists a compact set $\mathbb{X}_{1} \subset \mathbb{X}$ such that all optimal trajectories $x_{x, N}^{\star}$ with arbitrarily initial value $x \in \mathbb{X}_{0}$ and horizon $N \in \mathbb{N}$ satisfy $x_{x, N}^{\star}(N) \in \mathbb{X}_{1}$. In order to prove this property we use that Remark 2.2(iv) with $K=\mathbb{X}_{0}$ implies the inequality

$$
V_{N}(x) \leq N \ell\left(x^{e}, u^{e}\right)+C_{K}
$$


for all $x \in \mathbb{X}_{0}$. Let $u_{x, N}^{\star}$ be the optimal control corresponding to the optimal trajetory $x_{x, N}^{\star}$. Then from (2.4) and the definition of $\tilde{\ell}$ in (2.3) we obtain

$$
\begin{aligned}
V_{N}(x) & =\sum_{k=0}^{N-1} \ell\left(x_{x, N}^{\star}, u_{x, N}^{\star}\right)=\sum_{k=0}^{N-1} \tilde{\ell}\left(x_{x, N}^{\star}, u_{x, N}^{\star}\right)-\lambda(x)+\lambda\left(x_{x, N}^{\star}\right)+N \ell\left(x^{e}, u^{e}\right) \\
& \geq \alpha\left(\left\|x_{x, N}^{\star}(N-1)-x^{e}\right\|\right)+u_{x, N}^{\star}(N-1)^{T} R u_{x, N}^{\star}(N-1)+C_{1}+C_{2}+N \ell\left(x^{e}, u^{e}\right),
\end{aligned}
$$

where $C_{1}=\inf _{x \in \mathbb{X}_{0}}-\lambda(x)$ and $C_{2}=\inf _{x \in \mathbb{X}} \lambda(x)$. This implies that

$$
\alpha\left(\left\|x_{x, N}^{\star}(N-1)-x^{e}\right\|\right)+u_{x, N}^{\star}(N-1)^{T} R u_{x, N}^{\star}(N-1) \leq C_{K}-C_{1}-C_{2},
$$

i.e., particularly the norms of $x_{x, N}^{\star}(N-1)$ and (because of $R>0$ ) $u_{x, N}^{\star}(N-1)$ are bounded with a bound independent of $x$ and $N$. This implies that also the norm of $x_{x, N}^{\star}(N)=A x_{x, N}^{\star}(N-1)+B u_{x, N}^{\star}(N-1)$ is bounded independent of $x$ and $N$. Let $R_{1}>0$ be such a bound. Then, for $\mathbb{X}_{1}=B_{R_{1}}(0) \cap \mathbb{X}$ the relation $x_{x, N}^{\star}(N) \in \mathbb{X}_{1}$ holds for all $x \in \mathbb{X}_{0}$ and $N \in \mathbb{N}$ and the assertion follows.

We note that for some of the results in this section converse statements were obtained in [10], even for general nonlinear-nonquadratic optimal control problems. We will come back to this topic in Section 7, where we will present stronger results than those in [10] for the present linear-quadratic setting.

\section{A matrix condition for strict (pre)-dissipativity}

In this section we give necessary and sufficient conditions for strict (pre-)dissipativity in terms of matrix inequalities.

Lemma 4.1: Given $P \in \mathbb{R}^{n \times n}$, there exists $q \in \mathbb{R}^{n}$ such that LQ problem is strictly predissipative with storage function $\lambda(x)=x^{T} P x+q^{T} x$ if and only if the matrix inequality

$$
Q+P-A^{T} P A>0
$$

is satisfied. Particularly, if the problem is strictly pre-dissipative for certain $s, v$ and $c$, then the problem is strictly pre-dissipative for all $c, s$ and $v$. Moreover, if $P$ is positive definite then the problem is strictly dissipative.

Proof. We recall that strict pre-dissipativity holds if and only if the inequality (2.4), i.e., $\tilde{\ell}(x, u) \geq \alpha\left(\left\|x-x^{e}\right\|\right)$ holds for all $x \in X$ and $u \in U$ and the modified cost function $\tilde{\ell}$ from (2.3).

Now assume that the system is strictly pre-dissipative with $\lambda$ from the assumption. Then a straightforward computation yields that $\tilde{\ell}$ is of the form

$$
\tilde{\ell}(x, u)=x^{T}\left(Q+P-A^{T} P A\right) x+R(x, u),
$$

where $R(x, u)$ collects remainder terms which are linear or constant in $x$. Now inequality (2.4) together with $\tilde{\ell}\left(x^{e}, u^{e}\right)=0$ implies that $x \mapsto \tilde{\ell}\left(x, u^{e}\right)$ has a strict local minimum 
in $x=x^{e}$. For a function of the form (4.2) this is only possible if the quadratic part is strictly convex, i.e, if $Q+P-A^{T} P A$ is positive definite.

Conversely, assume $Q+P-A^{T} P A>0$. For a given $\gamma \in(0,1]$, set $P_{\gamma}:=\gamma P$ and

$$
Q_{\gamma}:=Q+P_{\gamma}-A^{T} P_{\gamma} A
$$

which is positive definite since $Q_{\gamma}=(1-\gamma) Q+\gamma\left(Q+P-A^{T} P A\right)>0$. Consider the modified stage cost

$$
\ell_{\gamma}(x, u):=\ell(x, u)+x^{T} P_{\gamma} x-f(x, u)^{T} P_{\gamma} f(x, u) .
$$

We claim that $\ell_{\gamma}$ is strictly convex in $(x, u)$, for a suitable value of $\gamma$. Indeed,

$$
\ell_{\gamma}(x, u)=x^{T} Q_{\gamma} x+u^{T}\left(R-B^{T} P_{\gamma} B\right) u-x^{T} A^{T} P_{\gamma} B u-u^{T} B^{T} P_{\gamma} A x+R(x, u),
$$

where $R(x, u)$ contains lower order terms in $(x, u)$. Setting $R_{\gamma}:=R-B^{T} P_{\gamma} B$ and $C:=$ $-A^{T} P B-B^{T} P A$, convexity of $\ell_{\gamma}$ is equivalent to positive definiteness of the matrix

$$
H:=\left(\begin{array}{cc}
2 Q_{\gamma} & \gamma C \\
\gamma C & 2 R_{\gamma}
\end{array}\right)
$$

Since $R_{\gamma}$ is positive definite for $\gamma$ sufficiently small, $H$ is positive definite if and only if the Schur complement of $R_{\gamma}$ in $H$, that is, $S_{\gamma}:=2 Q_{\gamma}-\gamma^{2} C\left(2 R_{\gamma}\right)^{-1} C$, is positive definite. Since $R_{0}=R$ is positive definite, the convergence $R_{\gamma}^{-1} \rightarrow R^{-1}$ as $\gamma \searrow 0$ follows, thus the norm of $\left(2 R_{\gamma}\right)^{-1}$ stays uniformly bounded for decreasing values of $\gamma$. Moreover, the Schur complement

$$
S_{\gamma}=Q_{\gamma}+(1-\gamma) Q+\gamma\left(Q+P-A^{T} P A-\gamma C\left(2 R_{\gamma}\right)^{-1} C\right)
$$

is positive definite for sufficiently small $\gamma$, since it is the sum of positive definite and positive semidefinite terms. We thus conclude that, for a sufficiently small $\bar{\gamma} \in(0,1]$, the modified stage cost $\ell_{\bar{\gamma}}$ is strictly convex in $(x, u)$. Then the optimal equilibrium problem

$$
\min _{x \in X, u \in U} \ell \bar{\gamma}(x, u), \quad \text { s.t. } \quad x-A x-B u=0,
$$

admits a unique global solution $\left(x^{e}, u^{e}\right)$. Applying [6, Proposition 4.3], we deduce the existence of a vector $q \in \mathbb{R}^{n}$ such that the LQ-problem with stage cost $\ell_{\bar{\gamma}}$ is strictly pre-dissipative at $\left(x^{e}, u^{e}\right)$ with storage function $\hat{\lambda}(x)=q^{T} x$. This implies that the LQproblem with the original stage cost $\ell$ is strictly pre-dissipative with storage function $\lambda(x)=$ $x^{T} P_{\bar{\gamma}} x+\hat{\lambda}(x)=x^{T} P_{\bar{\gamma}} x+q^{T} x$, which proves the claim.

The assertion on $c, s$ and $v$ follows immediately because the matrix condition is independent of $c, s$ and $v$ and also of $x^{e}$ and $u^{e}$, which implicitly depend on $c, s$ and $v$. Finally, positive definiteness of $P$ implies that the storage function $\lambda(x)=x^{T} P x+q^{T} x$ is bounded from below on the whole $\mathbb{R}^{n}$, hence the problem is dissipative.

Remark 4.2: The continuous time counterpart of the strict (pre-)dissipativity condition is

$$
\lambda(x(t)) \leq \lambda(x(0))+\int_{0}^{t} \ell(x(t), u(t))-\ell\left(x^{e}, u^{e}\right)-\alpha\left(\left\|x(t)-x^{e}\right\|\right) d t
$$


or, in differential form (provided $\lambda$ is $C^{1}$ )

$$
D \lambda(x) f(x, u) \leq \ell(x, u)-\ell\left(x^{e}, u^{e}\right)-\alpha\left(\left\|x-x^{e}\right\|\right) .
$$

For $\lambda$ of the form $\lambda(x)=x^{T} P x+q^{T} x$ and $f(x, u)=A x+B u$ this inequality becomes

$$
x^{T} A^{T} P x+x^{T} P A x+R_{1}(x, u)<x^{T} Q x+R_{2}(x, u),
$$

where $R_{1}$ and $R_{2}$ collect terms which are linear or constant in $x$. Similar to the arguments in the proof of Lemma 4.1 one then sees that strict pre-dissipativity is equivalent to the matrix inequality

$$
Q-A^{T} P-P A>0
$$

which thus replaces (4.1) in the continuous time setting. We conjecture that all subsequent results can be straightforwardly adapted to this equation.

\section{$5 \quad$ Observable and non-observable systems}

In this section we reformulate the matrix inequality (4.1) in terms of observability of $(A, C)$ in combination with eigenvalue conditions on $A$.

Definition 5.1: Consider a matrix pair $(A, C)$ with $A \in \mathbb{R}^{n \times n}, C \in \mathbb{R}^{l \times n}$. (i) We call $x_{0} \in \mathbb{R}^{n} \backslash\{0\}$ unobservable, if the solutions of $x(k+1)=A x(k)$ with $x(0)=x_{0}$ satisfy $C x(k)=0$ for all $k \in \mathbb{N}_{0}$. Otherwise we call $x_{0}$ observable.

(ii) We say that the matrix pair $(A, C)$ is observable, if every $x_{0} \in \mathbb{R}^{n} \backslash\{0\}$ is observable.

(iii) Let $x_{0}=w+i v \in \mathbb{C}^{n} \backslash\{0\}$ be an eigenvector of $A$ corresponding to the eigenvalue $\mu$. We say that $x_{0}$ is an unobservable eigenvector if either $w$ or $v$ is unobservable with respect of $(A, C)$. In this case, we call $\mu$ an unobservable eigenvalue.

One can show (for details see, e.g., [15, Chapter 6]) that $x_{0}$ is not observable if and only if it lies in the kernel of the observability matrix $\mathbf{O}(A, C):=\left(C^{T},(C A)^{T}, \ldots,\left(C A^{n-1}\right)^{T}\right)^{T}$. This implies that $(A, C)$ is observable if and only if the observability matrix has full rank. Another condition equivalent to observability is the Hautus-Criterion, which demands that the matrix

$$
\left(\begin{array}{c}
A-\mu I \\
C
\end{array}\right)
$$

has full rank for all eigenvalues $\mu$ of $A$.

Remark 5.2: If $x_{0}=w+i v \in \mathbb{C}^{n} \backslash\{0\}$ is an eigenvector of $A$ corresponding to the complex eigenvalue $\mu=a+i b$ with $b \neq 0$, then both $w$ and $v$ belong to $\mathbb{R}^{n} \backslash\{0\}$. Moreover, since $C$ is real, if $x_{0} \in \mathbb{C}^{n} \backslash\{0\}$ is an unobservable eigenvector of $A$ with eigenvalue $\mu$, then its complex conjugate $\bar{x}_{0}=w-i v$ is also an unobservable eigenvector of $A$ corresponding to the eigenvalue $\bar{\mu}$. Finally, from relations (A.3) in the Appendix we deduce that $w$ is observable if and only if $v$ is observable. Therefore, if $\mu$ is an unobservable eigenvalue then both $w$ and $v$ are unobservable.

Remark 5.3: Let $x_{0}$ be an unobservable real eigenvector or of the form $x_{0}=w$ for an unobservable complex eigenvector $w+i v$. For any $\gamma \in \mathbb{R}$ and $u \in \mathbb{U}$, the solution $x_{u}\left(k, \gamma x_{0}\right)$ is of the form

$$
x_{u}\left(k, \gamma x_{0}\right)=\gamma A^{k} x_{0}+x_{u}(k, 0), \quad \forall k \in \mathbb{N}_{0}
$$


Since $C A^{k} x_{0}=0$ for all $k \in \mathbb{N}_{0}$ this implies

$$
\begin{aligned}
& \ell\left(x_{u}\left(k, \gamma x_{0}\right), u\right) \\
& =x_{u}\left(k, \gamma x_{0}\right)^{T} Q x_{u}\left(k, \gamma x_{0}\right)+u(k)^{T} R u(k)+s^{T} x_{u}\left(k, \gamma x_{0}\right)+v^{T} u(k)+c \\
& =x_{u}(k, 0)^{T} Q x_{u}(k, 0)+u(k)^{T} R u(k)+s^{T} \gamma A^{k} x_{0}+s^{T} x_{u}(k, 0)+v^{T} u(k)+c \\
& =[\underbrace{x_{u}(k, 0)^{T} Q x_{u}(k, 0)+u(k)^{T} R u(k)+s^{T} x_{u}(k, 0)+v^{T} u(k)}_{=: \ell_{1}(k, u(0), \ldots, u(k))}]+[\underbrace{s^{T} \gamma A^{k} x_{0}+c}_{=: \ell_{2}\left(k, \gamma x_{0}\right)}] .
\end{aligned}
$$

From the last expression one sees that the stage cost decomposes into a first part $\ell_{1}$ which is independent of $x_{0}$ and $\gamma$ and a second part $\ell_{2}$ which is independent of $u$. Hence, the same holds for the optimization objective which can thus be written as

$$
J_{N}\left(\gamma x_{0}, u\right)=\sum_{k=0}^{N-1} \ell\left(x_{u}\left(k, \gamma x_{0}\right), u(k)\right)=\sum_{k=0}^{N-1} \ell_{1}(k, u(0), \ldots, u(k))+\sum_{k=0}^{N-1} \ell_{2}\left(k, \gamma x_{0}\right) .
$$

This implies that the optimal control $u^{*}$ is independent of $\gamma$ - unless maintaining the state constraints requires a change in the control action when $\gamma$ changes.

The following relations between observability and spectral properties of $A$, respectively, and the solvability of (4.1) follow from an adaptation of an argument that can be found in the literature.

Lemma 5.4: Consider the LQ-problem (2.1), (2.2) with $Q=C^{T} C$ and $(A, C)$ detectable. Then there exists a symmetric and positive definite matrix $P$ such that (4.1) holds.

Proof. We follow the ideas of [5, Lemma 1.7.3]. By duality, the detectability of $(A, C)$ is equivalent to the stabilizability of the pair $\left(A^{T}, C^{T}\right)$. Thus, there exists a matrix $F \in \mathbb{R}^{n \times n}$ such that $A^{T}+C^{T} F$ is asymptotically stable, i.e, there exists a symmetric and positive definite matrix $X$ such that

$$
\left(A^{T}+C^{T} F\right) X\left(A^{T}+C^{T} F\right)^{T}-X<0 .
$$

In particular, for nonzero $x \in \operatorname{Ker}(C)$, this implies that $x^{T} A^{T} X A x-x^{T} X x<0$. Then $Y:=A^{T} X A-X$ satisfies $Y<0$ on $\operatorname{Ker}(C)$. Let $\alpha>0$ and $U=\left[U_{1} U_{2}\right] \in \mathbb{R}^{n \times n}$ be a unitary matrix such that the columns of $U_{1}$ span $\operatorname{Ker}(C)$, and the relations $U_{1}^{T} Y U_{1}<0$, $U_{2}^{T} C^{T} C U_{2}>0$ hold. Then

$$
U^{T}\left(\alpha Y-C^{T} C\right) U=\left(\begin{array}{cc}
\alpha U_{1}^{T} Y U_{1} & \alpha U_{1}^{T} Y U_{2} \\
\alpha U_{2}^{T} Y U_{1} & \alpha U_{2}^{T} Y U_{2}-U_{2}^{T} C^{T} C U_{2}
\end{array}\right) .
$$

Since $\alpha U_{1}^{T} Y U_{1}<0$, the matrix $U^{T}\left(\alpha Y-C^{T} C\right) U$ is negative definite if its Schur complement

$$
-U_{2}^{T} C^{T} C U_{2}+\alpha\left(U_{2}^{T} Y U_{2}-U_{2}^{T} Y U_{1}\left(U_{1}^{T} Y U_{1}\right)^{-1} U_{1}^{T} Y U_{2}\right)
$$

is negative definite, which is true for $\alpha$ sufficiently small. For this appropriate choice of $\alpha$ we then conclude that $P:=\alpha X$ is a symmetric and positive definite solution to (4.1).

As a complementary result to Lemma 5.4, the following result extends [14, Theorem 23.7] to the case of $Q \geq 0$. We denote by $\sigma(A)$ the spectrum of the matrix $A$, and by $\mathbb{C}_{=1}$ the unit circle in the complex plane. 
Lemma 5.5: Consider the LQ-problem (2.1), (2.2). Assume that $A$ does not have eigenvalues $\mu$ with $|\mu|=1$. Then there exists a symmetric matrix $P$ solution to (4.1), which is positive definite if $|\mu|<1$ holds for all eigenvalues $\mu$ of $A$.

Proof. Thanks to Lemma A.1 in [6], the condition $\sigma(A) \cap \mathbb{C}_{=1}=\emptyset$ implies the existence of a symmetric matrix $P$ satisfying $P-A^{T} P A>0$. Since $Q \geq 0$, the inequality (4.1) admits $P$ as solution. Moreover, from the proof of Lemma A.1 in [6] we deduce that $P$ is positive definite if all eigenvalues of $A$ have norm (strictly) less than one.

\section{Eigenvalue conditions for strict (pre-)dissipativity}

The following result combines Lemma 5.4 and 5.5 to provide if-and-only-if conditions for strict (pre-)dissipativity.

Theorem 6.1: Consider the LQ-problem (2.1), (2.2) with $Q=C^{T} C$. Then the following holds:

(i) The problem is strictly dissipative if and only if $A$ does not have unobservable eigenvalues $\mu$ with $|\mu| \geq 1$.

(ii) The problem is strictly pre-dissipative if and only if $A$ does not have unobservable eigenvalues $\mu$ with $|\mu|=1$.

In both cases, the storage function can be chosen of the form $\lambda(x)=x^{T} P x+q^{T} x$, for suitable $P \in \mathbb{R}^{n \times n}$ and $q \in \mathbb{R}^{n}$.

Proof. Since all properties under consideration are invariant under coordinate changes, by Eq. (6.8) in [15] we may assume that $A$ and $C$ are of the form

$$
A=\left(\begin{array}{cc}
A_{1} & 0 \\
A_{3} & A_{2}
\end{array}\right), \quad C=\left(C_{1} 0\right)
$$

with $A_{1} \in \mathbb{R}^{r \times r}, A_{2} \in \mathbb{R}^{(n-r) \times(n-r)}, A_{3} \in \mathbb{R}^{(n-r) \times r}, C_{1} \in \mathbb{R}^{l \times r}, r \in\{0, \ldots, n\}$ being the rank of the observability matrix $\mathbf{O}(A, C)$, and $\left(A_{1}, C_{1}\right)$ being observable. Then $Q=C^{T} C$ is of the form

$$
Q=\left(\begin{array}{cc}
Q_{1} & 0 \\
0 & 0
\end{array}\right)
$$

with $Q_{1}=C_{1}^{T} C_{1} \in \mathbb{R}^{r \times r}$. We may thus apply Lemma 5.4 in order to obtain a symmetric and positive definite matrix $P_{1} \in \mathbb{R}^{r \times r}$ such that $Q_{1}+P_{1}-A_{1}^{T} P_{1} A_{1}>0$.

(a) Now assume that one of the eigenvalue conditions in (i) or (ii) holds. Since all unobservable eigenvectors of $A$ must be eigenvectors of $A_{2}$, we obtain that $A_{2}$ does not have eigenvalues $\mu$ with $|\mu|=1$. Hence, we may apply Lemma 5.5 to $A=A_{2}$ and $Q=0$ in order to obtain a symmetric matrix $P_{2} \in \mathbb{R}^{(n-r) \times(n-r)}$ with $P_{2}-A_{2}^{T} P_{2} A_{2}>0$. Here, $P_{2}$ is positive definite in case the eigenvalue condition from (i) holds.

For $\alpha>0$, define $P_{\alpha}:=\operatorname{diag}\left(P_{1}, \alpha P_{2}\right) \in \mathbb{R}^{n \times n}$. A straightforward computation yields

$$
Q+P_{\alpha}-A^{T} P_{\alpha} A=\left(\begin{array}{ll}
Q_{1}+P_{1}-A_{1}^{T} P_{1} A_{1}-\alpha A_{3}^{T} P_{2} A_{3} & -\alpha A_{3}^{T} P_{2} A_{2} \\
-\alpha A_{2}^{T} P_{2} A_{3} & \alpha P_{2}-\alpha A_{2}^{T} P_{2} A_{2}
\end{array}\right) .
$$


Since $Q_{1}+P_{1}-A_{1}^{T} P_{1} A_{1}>0$, the matrix $D_{\alpha}=Q_{1}+P_{1}-A_{1}^{T} P_{1} A_{1}-\alpha A_{3}^{T} P_{2} A_{3}$ is invertible for all $\alpha>0$ sufficiently small. Hence we can conclude that $Q+P_{\alpha}-A^{T} P_{\alpha} A$ is positive definite if its Schur complement

$$
\alpha P_{2}-\alpha A_{2}^{T} P_{2} A_{2}-\alpha^{2} A_{2}^{T} P_{2} A_{3} D_{\alpha}^{-1} A_{3}^{T} P_{2} A_{2}
$$

is so. Since $D_{0}$ is invertible, we deduce that $D_{\alpha}^{-1} \rightarrow D_{0}^{-1}$ as $\alpha \searrow 0$. Moreover, since $P_{2}-A_{2}^{T} P_{2} A_{2}$ is positive definite, the Schur complement is positive definite whenever $\alpha>0$ is sufficiently small. Fixing such a sufficiently small $\alpha>0$ and setting $P=P_{\alpha}$ we can apply Lemma 4.1 in order to conclude strict dissipativity if $P>0$, i.e., in case (i), and strict pre-dissipativity in case (ii).

(b) Conversely, assume that the system is strictly dissipative at an equilibrium $\left(x^{e}, u^{e}\right)$ and that the eigenvalue condition in (i) does not hold. Thus, let $\phi \in \mathbb{C}^{n} \backslash\{0\}$ be an unobservable eigenvector with eigenvalue $\mu$ satisfying $|\mu| \geq 1$. Let $w$ denote the real part of $\phi$, and set $x_{0}=x^{e}+\gamma w$ for some $\gamma \in \mathbb{R}$ that will be specified below. Consider the solution $x_{u}\left(k, x_{0}\right)$ corresponding to some control $u \in \mathbb{U}$. Thanks to the linearity of the dynamics and relation (5.1), $x(k)$ is decomposed as

$$
x_{u}\left(k, x_{0}\right)=x_{u}\left(k, x^{e}\right)+x_{0}(k, \gamma w)=x_{u}\left(k, x^{e}\right)+\gamma A^{k} w .
$$

In particular, for $u=u^{e}$, we have that

$$
x(k):=x_{u^{e}}\left(k, x_{0}\right)=x^{e}+\gamma A^{k} w .
$$

In case of $\mu$ real, we have that $\left\|x(k)-x^{e}\right\|=|\gamma||\mu|^{k}\|\phi\|$; in case of $\mu$ complex, we can appeal to the estimate from below in (A.2) that yields the existence of a constant $m>0$ such that $\left\|x(k)-x^{e}\right\|=|\gamma|\left\|A^{k} w\right\| \geq|\gamma||\mu|^{k} m$. Thus in both cases we can choose $|\gamma|$ sufficiently large to ensure that there exists $\delta>0$ such that $\alpha\left(\left\|x(k)-x^{e}\right\|\right) \geq \delta$ for all $k \in \mathbb{N}_{0}$. On the other hand, the definition of unobservable eigenvectors implies the condition $Q A^{k} w=0$ for all $k \in \mathbb{N}_{0}$. Thus, we deduce that

$$
\begin{aligned}
\ell\left(x(k), u^{e}\right) & =x(k)^{T} Q x(k)+\left(u^{e}\right)^{T} R u^{e}+s^{T} x(k)+v^{T} u^{e}+c \\
& =\left(x^{e}\right)^{T} Q x^{e}+\left(u^{e}\right)^{T} R u^{e}+s^{T} x^{e}+s^{T} \gamma A^{k} w+v^{T} u^{e}+c \\
& =\ell\left(x^{e}, u^{e}\right)+\gamma s^{T} A^{k} w .
\end{aligned}
$$

We now choose the sign of $\gamma$ such that $\gamma s^{T} w \leq 0$. Then in the real case a straightforward computation and in the complex case the application of Lemma A(ii) yields that there exist arbitrarily large $k \in \mathbb{N}$ with

$$
\sum_{j=0}^{k-1} \gamma s^{T} A^{j} w \leq 0
$$

For these $k$, induction over the strict dissipativity inequality together with identity (6.1) and with the relation $\alpha\left(\left\|x(k)-x^{e}\right\|\right) \geq \delta$ implies

$$
\lambda(x(k)) \leq \lambda\left(x_{0}\right)+\sum_{j=0}^{k-1} \gamma s^{T} A^{j} w-k \delta \leq \lambda\left(x_{0}\right)-k \delta .
$$


Since this holds for $k \in \mathbb{N}$ arbitrarily large, this implies that $\lambda(x(k))$ becomes unbounded for $k \rightarrow \infty$ which contradicts the boundedness of $\lambda$ from below in the dissipativity assumption.

(c) Finally, assume that the eigenvalue condition in (ii) does not hold and assume the problem is strictly pre-dissipative. With the same construction as in point (b) we obtain

$$
\lambda(x(k)) \leq \lambda\left(x_{0}\right)-k \delta
$$

for arbitrarily large $k \in \mathbb{N}$. If $x(k)=x_{0}$ holds for one of these $k$, this leads to the contradiction $\lambda\left(x_{0}\right) \leq \lambda\left(x_{0}\right)-k \delta$. In case of $x(k) \neq x_{0}$ for all these $k$, we obtain that $\lambda(x(k))$ is unbounded from below for $k \rightarrow \infty$. In order to contradicts the strict predissipativity assumption, we have to show that $(x(k))_{k}$ belongs to a bounded set. Indeed, in case of $\mu$ real, since $|\mu|=1$ we obtain $\left\|\gamma A^{k} w\right\|=|\gamma|\|w\|$ for all $k \in \mathbb{N}_{0}$, and thus $x(k)$ is contained in the closed ball centered at $x^{e}$ with radius $|\gamma|\|w\|$. A similar argument holds in the case of $\mu$ complex, since from Lemma A(i) there exists $M>0$ such that $\left\|\gamma A^{k} w\right\| \leq M$ for every $k \in \mathbb{N}_{0}$, thus $x(k)$ is contained in the closed ball centered at $x^{e}$ with radius $M$, and $\lambda(x(k))$ is unbounded from below in this bounded set.

\section{Turnpike implies strict (pre-)dissipativity}

Using the results developed so far, we can now state and prove converse results to those from Section 3.

Theorem 7.1: Consider the LQ-problem (2.1), (2.2) with $Q=C^{T} C$ and state and control constraint sets $\mathbb{X} \subseteq \mathbb{R}^{n}$ and $\mathbb{U} \subset \mathbb{R}^{m}$. Let $\left(x^{e}, u^{e}\right) \in \mathbb{X} \times \mathbb{U}$ be an equilibrium. Then the following holds:

(i) If $\mathbb{X}=\mathbb{R}^{n}$ and the problem has the turnpike property at $\left(x^{e}, u^{e}\right)$, then it is strictly dissipative at $\left(x^{e}, u^{e}\right)$.

(ii) If $\mathbb{X} \times \mathbb{U}$ contains a ball around $\left(x^{e}, u^{e}\right)$ and the problem has the near equilibrium turnpike property at $\left(x^{e}, u^{e}\right)$, then it is strictly pre-dissipative at $\left(x^{e}, u^{e}\right)$.

Proof. (i) We show the property by contraposition, i.e., we show that if strict dissipativity does not hold and $\mathbb{X}=\mathbb{R}^{n}$, then the turnpike property cannot hold. To this end, assume strict dissipativity does not hold. Then by Theorem 6.1 there exists an unobservable eigenvalue $\mu$ with $|\mu| \geq 1$. Let $w+i v$ be the corresponding eigenvector and set $x_{0}=w$ ( $v$ is possibly zero in case $\mu$ is real). Since for $\mathbb{X}=\mathbb{R}^{n}$ all solutions are feasible, from the discussion before the theorem we know that the optimal control $u^{*}$ for initial condition $\lambda x_{0}$ is independent of $\lambda \in \mathbb{R}$. The explicit solution formula (5.1) implies that for $\lambda_{1} \neq \lambda_{2}$ and all $k \in \mathbb{N}$ and $u \in \mathbb{U}^{k}$ the (in)equalities

$$
\left\|x_{u}\left(k, \lambda_{1} x_{0}\right)-x_{u}\left(k, \lambda_{2} x_{0}\right)\right\|=\left|\lambda_{1}-\lambda_{2}\right|\left\|A^{k} x_{0}\right\| \geq\left|\lambda_{1}-\lambda_{2}\right| C
$$

hold, where $C=\|w\|$ if $\mu$ is real and $C=m>0$ from Lemma A(i) otherwise. Since this in particular holds for the optimal controls, the turnpike property can hold for at most one of the two initial conditions. This contradicts Definition 2.1 which demands the property for all initial conditions in a bounded set. 
(ii) Again, we show the implication by contraposition. Assume that strict pre-dissipativity does not hold. Then by Theorem 6.1 there exists an unobservable eigenvalue $\mu$ with $|\mu|=1$. Let $w+i v$ be the corresponding eigenvector and set $x_{0}=x^{e}+\lambda w$ for $\lambda \in \mathbb{R}$. Then for the control $u \equiv u^{e}$ we obtain

$$
x_{u}\left(k, x_{0}\right)=x_{u}\left(k, x^{e}\right)+x_{0}(k, \lambda w)=x^{e}+\lambda A^{k} w .
$$

Since $\mu=1$ we obtain $\left\|A^{k} w\right\|=\|w\|$ if $\mu$ is real and otherwise

$$
m \leq\left\|A^{k} w\right\| \leq M
$$

for all $k \in \mathbb{N}$, with $M \geq m>0$ from Lemma A(i). Hence, since $\mathbb{X} \times \mathbb{U}$ contains a ball around $\left(x^{e}, u^{e}\right)$, for $|\lambda|$ sufficiently small we have that $x^{e}+\lambda A^{k} w$ lies in $\mathbb{X}$. Moreover, from (7.1) the same calculation as that for (5.2) (with $x^{e}$ replacing $x_{u}(k, 0)$ in $(5.1)$ ) leads to

$$
\ell\left(x_{u^{e}}\left(k, x_{0}\right)\right)=\ell\left(x^{e}, u^{e}\right)+\lambda s^{T} A^{k} w .
$$

Thus, choosing $|\lambda|$ sufficiently small and with appropriate sign such that $\lambda s^{T} w \leq 0$, from Lemma A(ii) we obtain that

$$
J_{N}(x, u)=N \ell\left(x^{e}, u^{e}\right)+\lambda \sum_{k=0}^{N-1} s^{T} A^{k} w \leq N \ell\left(x^{e}, u^{e}\right)
$$

for arbitrarily large $N$. However, because of (7.2) we obtain

$$
\left\|x_{u}\left(k, x_{0}\right)-x^{e}\right\|=\left\|\lambda A^{k} w\right\| \geq|\lambda| \min \{\|w\|, m\} \quad \forall k \in \mathbb{N}_{0} .
$$

This implies that the near optimal turnpike property does not hold.

\section{The main equivalence results}

In this section we combine the results of the previous sections in two theorems in order to obtain the "big picture", and we present one example for each situation. We start by considering the case without state constraints.

Theorem 8.1: Consider the LQ-problem (2.1), (2.2) with $(A, B)$ stabilizable, $Q=C^{T} C$ and state and control constraint sets $\mathbb{X}=\mathbb{R}^{n}$ and $\mathbb{U} \subseteq \mathbb{R}^{m}$. Then the following properties are equivalent

(i) The problem is strictly dissipative at an equilibrium $\left(x^{e}, u^{e}\right) \in \operatorname{int}(\mathbb{X} \times \mathbb{U})$.

(ii) The problem has the turnpike property at an equilibrium $\left(x^{e}, u^{e}\right) \in \operatorname{int}(\mathbb{X} \times \mathbb{U})$.

(iii) The pair $(A, C)$ is detectable, i.e., all unobservable eigenvalues $\mu$ of $A$ satisfy $|\mu|<1$.

Moreover, if one of these properties holds, then the equilibria in (i) and (ii) coincide and if $\mathbb{U}$ is convex then the exponential turnpike property holds. 
Proof. "(i) $\Rightarrow$ (ii)" follows from Corollary 3.2(i), "(ii) $\Rightarrow$ (i)" follows from Theorem 7.1(i), and "(i) $\Leftrightarrow$ (iii)" follows from Theorem 6.1(i). The fact that the equilibria coincide follows from Corollary 3.2 and the exponential turnpike property follows from Theorem 3.3.

We illustrate the application of this theorem by an example.

Example 8.2: Consider a linearized continuously stirred tank reactor model with two dimensional affine linear dynamics

$$
x(k+1)=\left(\begin{array}{cc}
0.853 & 0 \\
0.1065 & 0.9418
\end{array}\right) x(k)+\left(\begin{array}{c}
0.00457 \\
-0.00457
\end{array}\right) u(k)
$$

with $X=\mathbb{X}=\mathbb{R}^{2}, U=\mathbb{R}$ and control constraints $\mathbb{U}=[-10,10]$, and stage cost $\ell(x, u)=$ $\|x\|^{2}+0.05 u^{2}$. With the previous notations, we have that $Q=C=I$ is the identity matrix on $\mathbb{R}^{2}, R=0.05 I, s^{T}=v^{T}=c=0$, and

$$
A=\left(\begin{array}{cc}
0.853 & 0 \\
0.1065 & 0.9418
\end{array}\right), \quad B=\left(\begin{array}{c}
0.00457 \\
-0.00457
\end{array}\right)
$$

Then $(A, B)$ is stabilizable (but not controllable) and $(A, C)$ is detectable (as a matter of fact, observable), thus Theorem 8.1 implies that the system is strictly dissipative and has the exponential turnpike property at the equilibrium $\left(x^{e}, u^{e}\right)=(0,0)$, since $\mathbb{U}$ is convex. We mention that the strict dissipativity of the system also follows by [6, Proposition 4.3], and the exponential turnpike behaviour of the system can be deduced by [6, Theorem 6.2$]$.

The second theorem summarizes our results for compact state constraint set $\mathbb{X}$.

Theorem 8.3: Consider the LQ-problem (2.1), (2.2) with $Q=C^{T} C$ and state and control constraint sets $\mathbb{X} \subset \mathbb{R}^{n}$ compact and $\mathbb{U} \subseteq \mathbb{R}^{m}$. Then the following properties are equivalent

(i) The problem is strictly pre-dissipative at an equilibrium $\left(x^{e}, u^{e}\right) \in \operatorname{int}(\mathbb{X} \times \mathbb{U})$.

(ii) The problem has the near equilibrium turnpike property at an equilibrium $\left(x^{e}, u^{e}\right) \in$ $\operatorname{int}(\mathbb{X} \times \mathbb{U})$.

(iii) All unobservable eigenvalues $\mu$ of $A$ satisfy $|\mu| \neq 1$.

Moreover, if one of these properties holds, then the equilibria in (i) and (ii) coincide. If, in addition, $(A, B)$ is stabilizable then the turnpike property holds and if moreover $\mathbb{X} \times \mathbb{U}$ is convex then the exponential turnpike property holds.

Proof. "(i) $\Rightarrow$ (ii)" follows from Theorem 3.1(ii), "(ii) $\Rightarrow$ (i)" follows from Theorem 7.1(ii), and "(i) $\Leftrightarrow$ (iii)" follows from Theorem 6.1(ii). The fact that the equilibria coincide follows from Theorem 3.1(ii) and the exponential turnpike property follows from Theorem 3.3(ii).

Again, we illustrate the theorem by an example.

Example 8.4: Consider the control system $x(k+1)=2 x(k)+u(k)$ with $X=U=\mathbb{R}$, stage cost $\ell(x, u)=u^{2}$, and constraints $\mathbb{X}=[-0.5,0.5]$ and $\mathbb{U}=[-2,2]$. Since $\sigma(A) \cap \mathbb{C}_{=1}=\emptyset$, condition (iii) of Theorem 8.3 is satisfied, and it implies that the problem is strictly predissipative and has the near equilibrium turnpike property at the equilibrium $\left(x^{e}, u^{e}\right)=$ 
$(0,0)$. Moreover, since $A=2$ and $B=1$, and $\mathbb{X} \times \mathbb{U}$ is convex, the exponential turnpike property holds. Let us observe that, since $Q=0$ and thus $C=0$ and since $A$ has an unstable eigenvalue $\mu=2$, condition (iii) from Theorem 8.1 cannot be satisfied by this example, and hence in this case we shall rely on Theorem 8.3 which is tailored for problems with compact state constraint set $\mathbb{X}$. The strict dissipativity and the exponential turnpike property of this problem can also be deduced by [6, Theorem 4.5] and [6, Theorem 6.5], respectively.

Remark 8.5: Theorems 8.1 and 8.3 unify and relax [13, Theorem 1.1] and [6, Proposition 4.5] in the following sense: Theorem 1.1 in [13] demands observability of $(A, C)$ which implies that there are no unobservable eigenvalues, hence both Theorem 8.1(iii) and Theorem 8.3(iii) is implied. Proposition 4.5 in [6] excludes the existence of eigenvalues $\mu$ of $A$ with $|\mu|=1$, hence Theorem 8.3(iii) is implied.

An important feature that distinguishes the results in this paper from the results in $[6]$ and [13] is that Theorems 8.1 and 8.3 provide conditions which are also necessary and not merely sufficient. Hence, we can also detect the situations in which the turnpike property does not hold. Two final examples, both variants of Example 8.4, illustrate this fact.

Example 8.6: Consider again Example 8.4 but now without state constraints, i.e., with $\mathbb{X}=\mathbb{R}$. Now Theorem 8.3 is not applicable anymore, because $\mathbb{X}$ is no longer compact. On the other hand, the general assumptions of Theorem 8.1 are now satisfied but, as already observed in Example 8.4, condition (iii) of Theorem 8.1 is violated. Thus, we can conclude that the optimally controlled system does not exhibit the turnpike property. This is indeed the case, since for every initial condition it is obviously optimal to choose the optimal control $u \equiv 0$ for which the trajectories diverge to $\infty$ or $-\infty$.

Example 8.7: Consider again Example 8.4 but now with dynamics $x(k+1)=x(k)+u(k)$, i.e., with $A=1$. Since $Q=0$ and thus $C=0$, the single eigenvalue $\mu=1$ is unobservable, hence condition (iii) of Theorem 8.3 does not hold. Consequently, the near equilibrium turnpike property does not hold. This can also be checked directly: assume there is an equilibrium $\left(x^{e}, u^{e}\right) \in \mathbb{X} \times \mathbb{U}$ at which the near optimal turnpike property holds. Then from the equilibrium property we obtain $u^{e}=0$ and thus $\ell\left(x^{e}, u^{e}\right)=0$. Since for each initial value $x_{0} \in \mathbb{X}$ the control $u_{0} \equiv 0$ yields $J_{N}\left(x_{0}, u_{0}\right)=0$, we obtain $J_{N}\left(x_{0}, u_{0}\right) \leq \ell\left(x^{e}, u^{e}\right)$. However, the corresponding trajectories are constant, i.e., $x_{u_{0}}\left(k, x_{0}\right) \equiv x_{0}$, and thus violate the near optimal turnpike property at $\left(x^{e}, u^{e}\right)$ whenever $x_{0} \neq x^{e}$. This contradicts the fact that the near optimal turnpike property holds at the equilibrium $\left(x^{e}, u^{e}\right)$.

\section{A Appendix}

This appendix provides a technical lemma which was needed in several proofs throughout this paper.

Lemma A: Let $A \in \mathbb{R}^{n \times n}$ and $u=w+i v, w, v \in \mathbb{R}^{n}$, be a complex eigenvector of $A$ with eigenvalue $\mu=a+i b \in \mathbb{C}$ satisfying $b \neq 0$ and $|\mu| \geq 1$.

(i) Let $c_{0}, d_{0} \in \mathbb{R}$ such that $c_{0}^{2}+d_{0}^{2}=1$. Then

$$
A^{k}\left(c_{0} w+d_{0} v\right)=|\mu|^{k}\left(c_{k} w+d_{k} v\right) \quad \forall k \in \mathbb{N}_{0},
$$


with $c_{k}^{2}+d_{k}^{2}=1$. Moreover, there are constants $M \geq m>0$ such that for every $k \in \mathbb{N}_{0}$

$$
|\mu|^{k} m \leq\left\|A^{k}\left(c_{0} w+d_{0} v\right)\right\| \leq|\mu|^{k} M .
$$

(ii) Let $x(k):=A^{k} w$ and $s \in \mathbb{R}^{n}$ satisfying $s^{T} w \leq 0$. Then there exist arbitrarily large $k \in \mathbb{N}$ for which the inequality

$$
\sum_{j=0}^{k} s^{T} x(j) \leq 0
$$

holds.

Proof. For $r=|\mu|, \sigma=\ln r, \vartheta=\arg (\mu) \in[0,2 \pi)$ and $z=\sigma+i \vartheta$, i.e., $\mu=e^{\sigma}(\cos \vartheta+$ $i \sin \vartheta)=e^{z}$, the identity $A u=\mu u$ can be rewritten as $A u=e^{z} u$ which implies

$$
A^{k} u=e^{z k} u
$$

for all $k \in \mathbb{N}_{0}$. Note that $\vartheta \neq 0$ and $\vartheta \neq \pi$ because $b \neq 0$.

i) A straightforward computation gives that, for all $k \in \mathbb{N}_{0}$,

$$
A^{k} w=r^{k}(\cos (k \vartheta) w-\sin (k \vartheta) v), \quad A^{k} v=r^{k}(\sin (k \vartheta) w+\cos (k \vartheta) v),
$$

thus (A.1) holds for all $k \in \mathbb{N}_{0}$ with

$$
c_{k}=c_{0} \cos (k \vartheta)+d_{0} \sin (k \vartheta), \quad d_{k}=d_{0} \cos (k \vartheta)-c_{0} \sin (k \vartheta),
$$

thus $c_{k}^{2}+d_{k}^{2}=c_{0}^{2}+d_{0}^{2}=1$. In order to prove (A.2), observe that from (A.1) we obtain

$$
\left\|A^{k}\left(c_{0} w+d_{0} v\right)\right\|^{2}=|\mu|^{2 k}\left\|c_{k} w+d_{k} v\right\|^{2} .
$$

It thus suffices to show the existence of $M \geq m>0$ with $m^{2} \leq\|c w+d v\|^{2} \leq M^{2}$ for all $c, d \in \mathbb{R}$ with $c^{2}+d^{2}=1$. For the squared Euclidean norm it holds that

$$
\|c w+d v\|^{2}=c^{2}\|w\|^{2}+d^{2}\|v\|^{2}+2 c d\langle w, v\rangle
$$

Since $2\langle w, v\rangle \leq\|w\|^{2}+\|v\|^{2}$ and $|c| \leq 1$ and $|d| \leq 1$, we obtain the upper bound $M=$ $2\left(\|w\|^{2}+\|v\|^{2}\right)$.

In order to find the lower bound $m>0$, let $c_{*} \in \mathbb{R}, d_{*} \in \mathbb{R}$ with $c_{*}^{2}+d_{*}^{2}=1$ be such that

$$
\min _{c^{2}+d^{2}=1} c^{2}\|w\|^{2}+d^{2}\|v\|^{2}+2 c d\langle w, v\rangle=c_{*}^{2}\|w\|^{2}+d_{*}^{2}\|v\|^{2}+2 c_{*} d_{*}\langle w, v\rangle=: m .
$$

Clearly, this $m$ is a lower bound and it thus remains to show $m>0$. To this end, If either $c_{*}=0$ or $d_{*}=0$ the assertion follows because $m=\|v\|$ or $m=\|w\|$, respectively. Otherwise, we set $w_{*}=c_{*} w$ and $v_{*}=d_{*} v$. Then, since $w$ and $v$ span a two dimensional subspace (the sum of the eigenspaces corresponding to the complex conjugate eigenvalues $\mu$ and $\bar{\mu})$, we know from the Cauchy-Schwarz inequality that $\left|\left\langle w_{*}, v_{*}\right\rangle\right|<\left\|w_{*}\right\|\left\|v_{*}\right\|$, because equality can only hold if $w_{*}$ and $v_{*}$ and thus $w$ and $v$ are linearly dependent. This yields

$$
m=\left\|w_{*}\right\|^{2}+\left\|v_{*}\right\|^{2}+2\left\langle w_{*}, v_{*}\right\rangle>\left\|w_{*}\right\|^{2}+\left\|v_{*}\right\|^{2}-2\left\|w_{*}\right\|\left\|v_{*}\right\|=\left(\left\|w_{*}\right\|-\left\|v_{*}\right\|\right)^{2} \geq 0
$$


and thus the claim $m>0$.

ii) Since $s$ and $A$ are real, from $u=w+i v$ we obtain

$$
s^{T} A^{j} w=s^{T} \Re\left(A^{j}(w+i v)\right)=\Re\left(e^{z j} s^{T} u\right),
$$

which implies

$$
\sum_{j=0}^{k} s^{T} x(j)=s^{T} \sum_{j=0}^{k} A^{j} w=\Re\left(s^{T} \sum_{j=0}^{k} e^{z j} u\right) .
$$

Because of

$$
\int_{j}^{j+1} e^{z \tau} d \tau=\frac{e^{z(j+1)}-e^{z j}}{z}=e^{z j} \frac{e^{z}-1}{z}
$$

we can write

$$
s^{T} \sum_{j=0}^{k} e^{z j} u=s^{T} \frac{z}{e^{z}-1} \int_{0}^{k+1} e^{z \tau} d \tau u=\frac{e^{z(k+1)}-1}{e^{z}-1} s^{T} u .
$$

Setting $\varrho_{k}:=\frac{e^{z(k+1)}-1}{e^{z}-1} \in \mathbb{C}$, this yields

$$
\sum_{j=0}^{k} s^{T} x(j)=\Re\left(\varrho_{k}\right) s^{T} w-\Im\left(\varrho_{k}\right) s^{T} v .
$$

We thus have to show that the right-hand side of this expression is negative for arbitrarily large $k$. To this end, observe that for each $\varepsilon>0$ there exist arbitrarily large $k \in \mathbb{N}$ such that the relation

$$
k \vartheta \bmod 2 \pi \in[2 \pi-\varepsilon, 2 \pi) \cup[0, \varepsilon]
$$

holds: for $\vartheta / \pi$ being rational, i.e., equal to $p / q$ for $p \in \mathbb{Z}, q \in \mathbb{N}$ this is true whenever $k$ is an integer multiple of $2 q$, because in this case we have $k \vartheta \bmod 2 \pi=0$. In case $\vartheta / \pi$ is irrational, the claim follows from the equidistribution theorem for the sequence $a k \bmod 1$ with irrational $a$, see, e.g., [2].

One computes the numerators of $\Re\left(\varrho_{k}\right)$ and $\Im\left(\varrho_{k}\right)$ as

$$
[\cos (\vartheta) \cos ((k+1) \vartheta)+\sin (\vartheta) \sin ((k+1) \vartheta)] r^{k+2}-\cos ((k+1) \vartheta) r^{k+1}-\cos (\vartheta) r+1
$$

and

$$
[\cos (\vartheta) \sin ((k+1) \vartheta)-\sin (\vartheta) \cos ((k+1) \vartheta)] r^{k+2}-\sin ((k+1) \vartheta) r^{k+1}+\sin (\vartheta) r,
$$

respectively, while the denominator is always positive.

In case $r>1$, for large $k$ the term in $r^{k+2}$ becomes dominant in the expressions of $\Re\left(\varrho_{k}\right)$ and $\Im\left(\varrho_{k}\right)$, with coefficients

$$
a_{k}:=\cos (\vartheta) \cos ((k+1) \vartheta)+\sin (\vartheta) \sin ((k+1) \vartheta)=\cos (k \vartheta)
$$

and

$$
b_{k}:=\cos (\vartheta) \sin ((k+1) \vartheta)-\sin (\vartheta) \cos ((k+1) \vartheta)=\sin (k \vartheta)
$$


respectively. For $k \vartheta \bmod 2 \pi=0$ we deduce that $a_{k}=1$ and $b_{k}=0$, rendering the righthand side of (A.4) negative thanks to $s^{T} w \leq 0$. By continuity, the whole expression in the right-hand side of (A.4) is negative whenever $k \vartheta \bmod 2 \pi \in[2 \pi-\varepsilon, 2 \pi) \cup[0, \varepsilon]$, which by (A.5) holds true for arbitrarily large $k \in \mathbb{N}$.

In case $r=1$, the numerators of $\Re\left(\varrho_{k}\right)$ and $\Im\left(\varrho_{k}\right)$ in (A.6) and (A.7) become

$$
\operatorname{Num}\left(\Re\left(\varrho_{k}\right)\right)=\cos (k \vartheta)-\cos ((k+1) \vartheta)-\cos (\vartheta)+1
$$

and

$$
\operatorname{Num}\left(\Im\left(\varrho_{k}\right)\right)=\sin (k \vartheta)-\sin ((k+1) \vartheta)+\sin (\vartheta) .
$$

Then, for $k \vartheta \bmod 2 \pi=0$, we find out that $\cos (k \vartheta)=1, \sin (k \vartheta)=0, \cos ((k+1) \vartheta)=\cos (\vartheta)$ and $\sin ((k+1) \vartheta)=\sin (\vartheta)$, thus (A.8) and (A.9) give that $N u m\left(\Re\left(\varrho_{k}\right)\right)=2-2 \cos (\vartheta)>0$ since $\vartheta \neq 0$, and $\operatorname{Num}\left(\Im\left(\varrho_{k}\right)\right)=0$. We thus conclude that the right-hand side of (A.4) is negative and hence, by continuity, there is $\varepsilon>0$ such that the sign is preserved whenever $k \vartheta \bmod 2 \pi \in[2 \pi-\varepsilon, 2 \pi) \cup[0, \varepsilon]$. By (A.5) this is true for arbitrarily large $k \in \mathbb{N}$, which shows the claim.

Acknowledgement: We would like to thank Tobias Damm for his help with the proof of Lemma 5.4 and Enrique Zuazua for fruitful discussions on the subject.

\section{References}

[1] B. D. O. Anderson And P. V. Kokotović, Optimal control problems over large time intervals, Automatica, 23 (1987), pp. 355-363.

[2] G. D. Birkhoff, Proof of the ergodic theorem, Proc. Nat. Acad. Sci., 17 (1931), pp. 656-660.

[3] A. Boccia, L. Grüne, And K. Worthmann, Stability and feasibility of state constrained MPC without stabilizing terminal constraints, Systems Control Lett., 72 (2014), pp. 14-21.

[4] D. A. Carlson, A. B. Haurie, and A. Leizarowitz, Infinite horizon optimal control - Deterministic and Stochastic Systems, Springer-Verlag, Berlin, second ed., 1991.

[5] T. DAmm, Rational matrix equations in stochastic control, vol. 297 of Lecture Notes in Control and Information Sciences, Springer-Verlag, Berlin, 2004.

[6] T. Damm, L. Grüne, M. Stieler, and K. Worthmann, An exponential turnpike theorem for dissipative discrete time optimal control problems, SIAM J. Control Optim., 52 (2014), pp. 1935-1957.

[7] R. Dorfman, P. A. Samuelson, and R. M. Solow, Linear Programming and Economic Analysis, Dover Publications, New York, 1987. Reprint of the 1958 original.

[8] L. GRÜNE, Economic receding horizon control without terminal constraints, Automatica, 49 (2013), pp. 725-734. 
[9] L. GRÜnE, Approximation properties of receding horizon optimal control, Jahresber. DMV, 118 (2016), pp. 3-37.

[10] L. Grüne AND M. A. MÜLleR, On the relation between strict dissipativity and the turnpike property, Syst. Contr. Lett., 90 (2016), pp. 45-53.

[11] L. Grüne And J. Pannek, Nonlinear Model Predictive Control. Theory and Algorithms, Springer-Verlag, London, 2nd ed., 2017.

[12] L. W. MCKENZIE, Optimal economic growth, turnpike theorems and comparative dynamics, in Handbook of Mathematical Economics, Vol. III, North-Holland, Amsterdam, 1986, pp. 1281-1355.

[13] A. Porretta And E. Zuazua, Long time versus steady state optimal control, SIAM J. Control Optim., 51 (2013), pp. 4242-4273.

[14] W. J. Rugh, Linear system theory, Prentice Hall,, Upper Saddle River, N.J., 2nd ed. ed., 1996.

[15] E. D. Sontag, Mathematical Control Theory, Springer Verlag, New York, 2nd ed., 1998.

[16] E. TRÉlat And E. Zuazua, The turnpike property in finite-dimensional nonlinear optimal control, J. Differential Equations, 258 (2015), pp. 81-114.

[17] J. von Neumann, A model of general economic equilibrium, The Review of Economic Studies, 13 (1945), pp. 1-9.

[18] J. C. Willems, Least squares stationary optimal control and the algebraic Riccati equation, IEEE Trans. Autom. Control, 16 (1971), pp. 621-634.

[19] J. C. Willems, Dissipative dynamical systems. I. General theory, Arch. Rational Mech. Anal., 45 (1972), pp. 321-351.

[20] J. C. Willems, Dissipative dynamical systems. II. Linear systems with quadratic supply rates, Arch. Rational Mech. Anal., 45 (1972), pp. 352-393. 